\title{
Association between Dental Prosthesis and Periodontal Disease among Patients Visiting a Tertiary Dental Care Centre in Eastern Nepal.
}

Mansuri M, Shrestha A

Department of Public Health Dentistry

College of Dental Surgery,

BPKIHS, Dharan, Nepal

\section{Corresponding Author}

Mustapha Mansuri

Department of Public Health Dentistry

College of Dental Surgery,

BPKIHS, Dharan, Nepal

E-mail: mustapha.mansuri@bpkihs.edu

\section{Citation}

Mansuri M, Shrestha A. Association Between Dental Prosthesis and Periodontal Disease Among Patients Visiting a Tertiary Dental Care Centre in Eastern Nepal. Kathmandu Univ Med J 2015;51(3):200-3.

\begin{abstract}
Background

Dental caries and Periodontal diseases are the most prevalent oral health problems present globally. The distribution and severity of such oral health problems varies in different parts of the world and even in different regions of the same country. Nepal is one of the country with higher prevalence rate of these problems. These problems arise in association with multiple factors.
\end{abstract}

\section{Objective}

This study was carried out to describe the periodontal status and to analyse the association of periodontal disease with the wearing of fixed or removable partial dentures in a Nepalese population reporting to the College of Dental Surgery, B P Koirala Institute of Health Sciences, Dharan, Nepal.

\section{Method}

This study comprised of a sample of 200 adult individuals. All data were collected by performing clinical examinations in accordance with the World Health Organization Oral Health Surveys Basic Methods Criteria. It included the Community Periodontal Index and dental prosthesis examination.

\section{Result}

A descriptive analysis was performed and odds ratio (1.048) and $95 \%$ confidence interval $(1.001 ; 1.096)$ was found out. The mean age of the population participated in the study was $41.82 \pm 14.80$ years. A total of 93 (46.5\%) males and 107 (53.5\%) females participated in the study. Among these subjects, $100 \%$ presented some periodontal problems. The statistical analysis indicated that the probability of periodontal disease with regards to wearing partial dentures was not significant as suggested by the odds ratio (1.048).

\section{Conclusion}

There is no association of the wearing of dental prosthesis (RPD and/or FPD) with the periodontal disease and suggests a need for populations based oral health education programs, plaque control programs to reduce the incidence of periodontal disease.

\section{KEY WORDS}

Community periodontal index, dental prosthesis, oral health, periodontal disease. 


\section{INTRODUCTION}

Dental caries and periodontal diseases have been historically considered the most important global oral health burdens. ${ }^{1}$ At present, the distribution and severity of oral diseases vary in different parts of the world and within the same country or region. In Nepal, approximately $31 \%$ of population age $35-44$ years surveyed in a study had developed deep periodontal pockets. ${ }^{2}$

A global database was established and over a number of years an increasing number of oral epidemiological studies have been conducted applying World Health Organization (WHO) Oral Health Surveys basic methods and criteria., ${ }^{1,3}$ To assess the periodontal status of populations, the WHO recommended the Community Periodontal Index (CPI) application, which verifies the state of periodontal health in view of gingival bleeding, periodontal calculus and pocket. The CPI has been widely used to measure the level of periodontal diseases and treatment needs in populations, and it allows for international comparisons. ${ }^{1,3}$

Oral cavity harbours millions of micro-organisms. These micro-organisms can lead to various oral and systemic problems if proper oral hygiene is not maintained. Removable/fixed partial dentures provides shelter for micro-organisms. There is difficulty in maintaining proper oral hygiene among them. It can lead to gingival and periodontal diseases. Periodontal disease is marked by bacterial overgrowth. Dental prosthesis has got an influence on the periodontal conditions, the risk of caries and the amount of stress on natural teeth. ${ }^{1,4}$ Numerous studies have shown that there is deterioration of the gingival and periodontal conditions after dental prosthesis are fitted in. ${ }^{1,4,5}$ Studies suggest that the use of partial dentures may constitute a risk factor for periodontal health. ${ }^{1,4-8}$

Continuing surveillance of levels and patterns of risk factors of periodontal disease is of fundamental importance to planning and evaluating community preventive activities and oral health promotion. ${ }^{1}$ The purpose of this study is to describe the periodontal conditions and to analyze the association between the wearing of fixed or removable partial dentures and periodontal disease in adult population in Nepal.

\section{METHODS}

The study was carried out in B P Koirala Institute of Health Sciences, Dharan, starting from $24^{\text {th }}$ September, 2013 for about two months. This study comprised of a sample of 200 adult individuals. Data were collected by clinical examinations in accordance with the World Health Organization Oral Health Surveys Basic Methods and Criteria, ${ }^{1}$ which included the Community Periodontal Index and dental prosthesis examination.

Place and Duration of Study: B P Koirala Institute of Health Sciences, Dharan, Nepal and the duration of study was 2 months.
Inclusion criteria: peoples coming to BPKIHS dental OPD and department of Prosthodontics and who were willing to participate.

Exclusion criteria: handicapped, psychiatric illness (Schizophrenia, Alzheimer's disease, Bipolar disorder, Autism, Paranoid disorder) if present.

Ethical clearance: It was obtained from the Institutional Ethical Review Board of B P Koirala Institute of Health Sciences, Dharan, Nepal.

Data were entered into Microsoft Excel and statistical analysis was performed by using Social Package for Statistical Studies (SPSS 16) to determine the mean age, percentage distribution and the mean number of sextants for the descriptive study of periodontal parameters. Odds ratio and $95 \%$ confidence interval were calculated to study the association of dental prosthesis with periodontal disease.

\section{RESULTS}

We took a sample of randomly selected 100 patients who were not wearing the prosthesis and another 100 patients who were wearing either removable or fixed partial denture or both, reporting to the College of Dental Surgery, BPKIHS, Dharan, Nepal.

Table 1. Socio-demographic profile of respondents

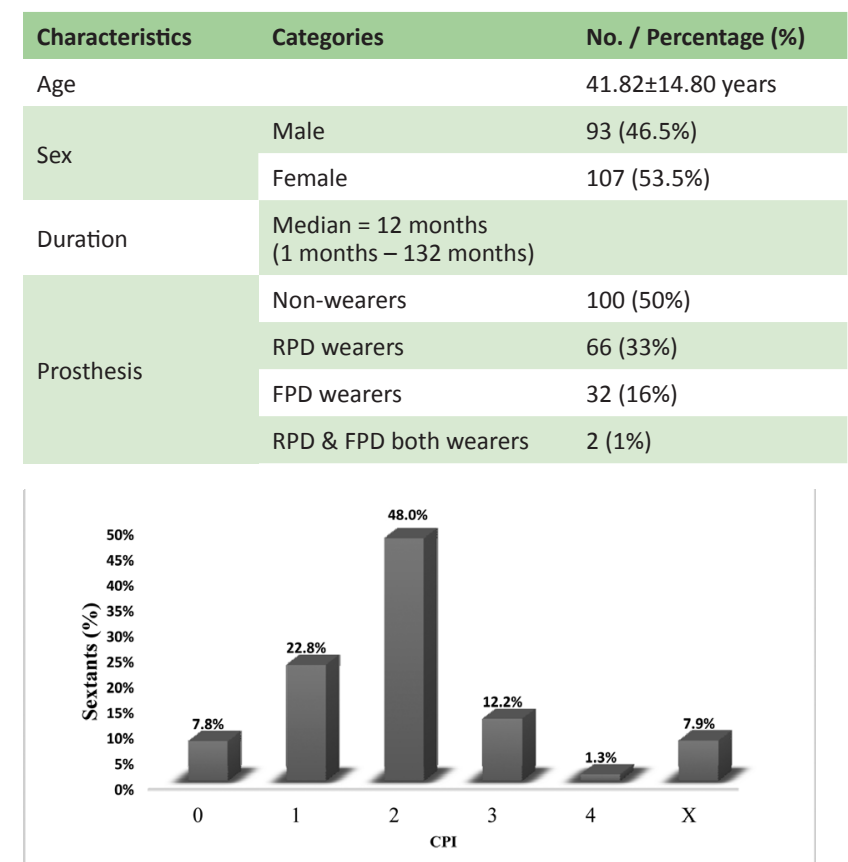

Figure 1. Percentage distribution of sextants by the highest Community Periodontal Index (CPI) score

Table 1 and Figure 1 show the prevalence of periodontal condition measured by the Community Periodontal Index (CPI) on the subjects who underwent the periodontal examination. The mean age of the population participated in the study was $41.82 \pm 14.80$ years. A total of 93 (46.5\%) males and 107 (53.5\%) females participated in the study. Among all, there were 100 (50\%) non-denture wearers, 66 (33\%) Removable partial Denture (RPD) wearers, 32 (16\%) 
Fixed Partial Denture wearers and 2 (1\%) both RPD \& FPD both wearers. Among these subjects, $100 \%$ presented some periodontal problems.

A mean number of 4.2 sextants presented bleeding and calculus. The mean number of sextants with healthy periodontal tissues was 0.5 , which represents $7.8 \%$ of the total. The sum of the conditions (score 1 and 2) represented $70.8 \%$ of total sextants which represents bleeding and calculus as worse condition, whereas 162 sextants were found to have a pocket of $4 \mathrm{~mm}$ or more, score 3 and 4 represented by $13.5 \%$ of sextants. Excluded sextant (score $x)$, due to their remaining less than 2 teeth, were less prevalent (7.9\%).

Table 2. Percentage distribution of subjects and mean number of sextants by the highest Community Periodontal Index (CPI) score.

\begin{tabular}{lllllllll|} 
& \multicolumn{10}{c|}{ CPI Score } \\
& 0 & 1 & 2 & 3 & 4 & X & 9 & Total \\
\hline \% Patients & 0 & 10 & 53.5 & 31 & 5.5 & 0 & - & 100 \\
$\begin{array}{l}\text { Mean no. of } \\
\text { Sextants }\end{array}$ & 0.5 & 1.4 & 2.8 & 0.7 & 0.1 & 0.5 & - & 6.0 \\
\hline
\end{tabular}

Table 2 shows that $53.5 \%$ of subjects (highest distribution) with mean number sextants 2.8 had CPI score of 2 and $31 \%$ of subjects with mean number sextant 0.7 had CPI score of 3. Others were distributed in lesser number of subjects: $10 \%$ with mean number sextant 1.4 and $5 \%$ of subjects with mean number sextants 0.1 had CPI score 1 and 4 respectively.

Table 3. Number and percentage distribution of sextants by the presence of periodontal disease and use of partial dentures.

\begin{tabular}{|llllll|l|l|}
\hline Prosthesis & \multicolumn{3}{c}{ Periodontal disease } & $\begin{array}{c}\text { Odds } \\
\text { ratio }\end{array}$ & $\begin{array}{l}\text { 95\% confidence } \\
\text { interval }\end{array}$ \\
& Present & Absent & Total & & & \\
\hline Present & 466 & 42 & 508 & & Lower & Upper \\
\hline Absent & 543 & 51 & 594 & 1.048 & & \\
\hline Total & 1009 & 93 & 1102 & & 1.001 & 1.096 \\
\hline
\end{tabular}

The statistical analysis indicated that the probability of periodontal disease with regards to wearing partial dentures was not significant as suggested by the odds ratio (1.048).

\section{DISCUSSION}

This study was done to address the hypothesis that there was no difference in periodontal conditions between nondenture wearers and removable or fixed partial denture wearers in eastern Nepalese adult population. The results of this analysis suggest that partial dentures do not play a very significant role in the development of periodontal disease.

In this study, all subjects presented signs of periodontal disease. But there is no much difference in the periodontal conditions among prosthesis wearers and non-wearers. This means that periodontal alterations were more generalized among both prosthesis wearers and non-wearers.

We usually expect worsening of periodontal conditions in prosthesis wearers than in non-wearers. But it was not found so in our study as both prosthesis wearers and non-wearers have similar periodontal conditions. The presence of prosthesis whether it is RPD or FPD, worsens the periodontal conditions among them. They usually retain food debris and make oral hygiene maintenance difficult leading to formation of plaque and calculus or even pockets in worse conditions. There are other factors like their frequent visits to the dentist, getting proper oral hygiene instructions from the dentist, frequent oral prophylaxis, and frequent follow ups which also play an important role. Among the factors that account for poor periodontal condition are the lack of professional advice regarding oral health maintenance and the low rate of follow-up. ${ }^{6}$ Whereas among non-wearers, the worsening periodontal condition what we found can be expected due to lack of all these factors.

To understand these differences, it is necessary to know the social determinants of health involved in the health-disease process of periodontal disease, like income, education, available health facilities, habitation conditions, work, transport, sanitation and environment. Various researches have shown that, ${ }^{6-8}$ on comparing the periodontal condition of abutment teeth and natural teeth, a statistically significant difference was found between both periodontal conditions, the abutment teeth presenting a greater depth of gingival crevice, an increase of calculus and dental mobility. It is believed that the increase in plaque accumulation is related to negligence of the patient and not necessarily brought about by the prosthesis. That periodontal alterations may be solely due to the patient's poor oral hygiene with no prosthesis involvement in this result. ${ }^{1}$

A research performed in rural and urban areas of a Brazilian city compared the oral health condition of these regions, including the main oral diseases as dental decay and periodontal disease, and the authors observed that rural residents had worse index than urban residents. ${ }^{9}$ Another study carried out in a rural area of São Paulo State, Brazil, found values as $99.5 \%$ of children aged 6 to 14 years presenting signs of periodontal disease. ${ }^{10}$ Despite some methodological differences among surveys performed with rural populations, it is distinguished the worse periodontal conditions of these populations. These studies suggest that there might be other factors associated with worsening of periodontal conditions among them such as low income, lack of education, lack of available health facilities in rural areas. Our study also casts doubts on association of wearing partial dentures with periodontal disease and indicate probability of other social determinants of health involved in the health-disease process of periodontal disease. 
For rural populations, the strategies must emphasize the importance of adapting the services to the necessities and difficulties of those populations. The access to health services must be facilitated so all can be assisted, not only in relation to curative treatment, but also, and mainly, to preventive and educative activities. Finally, the results highlight the need for further epidemiologic studies to clarify the relationship between dental prosthesis and periodontal disease, types of dentures and period of development of disease.

\section{CONCLUSION}

Thus, from this study it is concluded that there is no association of the wearing of dental prosthesis (RPD and/ or FPD) with the periodontal disease. The results suggest a need for populations based oral health education programs, plaque control programs in an attempt to reduce the incidence of development of periodontal disease among prosthesis non-wearers and wearers too.

\section{ACKNOWLEDGEMENT}

I would like to thank Assistant Professor, Mr. DD Baral, School of Public Health and Community Medicine, BPKIHS, Dharan for his help in statistical analysis.

\section{REFERENCES}

1. Suzely Adas Saliba Moimaz, Nemre Adas Saliba, Orlando Saliba, Lívia Guimarães Zina, Márcio Rogério Curtis Bolonhez; Association between dental prosthesis and periodontal disease in a rural Brazilian community. Brazilian Journal of Oral Sciences. 2006 OctoberDecember;19(5): 1226-31.

2. Ministry of Health and Population. National Oral Health Policy, Nepal; 2004. \{http://www.mohp.gov.np/english/files/new_publications/92-National-Oral-Health-Policy.pdf\}

3. World Health Organization. Oral health surveys: basic methods. 4th ed. Geneva: WHO; 1997.

4. Mojon P, Rentsch A, Budtz-Jørgensen E. Relationship between prosthodontic status, caries, and periodontal disease in a geriatric population.Int J Prosthodont. 1995 Nov-Dec; 8(6):564-71.

5. Kern $\mathrm{M}$, Wagner B. Periodontal findings in patients 10 years after insertion of removable partial dentures. J Oral Rehabil. 2001 Nov; 28(11):991-7.

6. Leles C R, Melo M, Oliveira M MM. Clinical evaluation of removable partial denture's effect on dental and periodontal condition of partially edentulous subjects. ROBRAC. 1999; 8:14-8.

7. Yusof Z, Isa Z. Periodontal status of teeth in contact with denture in removable partial denture wearers. J Oral Rehabil. 1994; 21: 77-86.

8. Zlataric D K, Celebic A, Valentic-Peruzovic M. The effect of removable partial dentures on periodontal health of abutment and nonabutment teeth. J Periodontol. 2002; 73: 137-44.

9. Cangussu MCT, Coelho EO, Castellanos FRA. Epidemiology and inequalities in oral health at 5, 12 and 15 years old in the city of in Itatiba, Säo Paulo, 2000. Rev FacOdontol Bauru. 2001; 9: 77-85.

10. MarcantonioJúnior E, Santos FA. Evaluation of conditions and periodontal treatment needs of rural schoolchildren in the Northwest of the Säo Paulo State. Rev Odontol UNESP. 1998; 27: 449-58. 